\title{
OBTENCIÓN DE BARRIDOS LÁSER 3D NIVELADOS CON EL ROBOT MÓVIL ANDÁBATA EN MOVIMIENTO
}

\author{
Jorge L. Martínez, Jesús Morales y Manuel Zafra \\ jlmartinez@uma.es, jesus.morales@uma.es, manuzagra@gmail.com \\ Universidad de Málaga, Andalucía Tech, Dpto. Ingeniería de Sistemas y Automática \\ Calle Dr. Ortiz Ramos s/n, 29071-Málaga
}

\begin{abstract}
Resumen
Este artículo describe la adquisición de barridos tridimensionales (3D) nivelados en el robot móvil Andábata sin necesidad de detener su movimiento. Para ello, la computadora de Andábata debe integrar cada uno de los rangos láser, adquiridos con unos determinados ángulos de cabeceo y guiñada, con la información odométrica y las medidas de inclinación del vehículo para producir coordenadas Cartesianas niveladas referenciadas al inicio de cada barrido. Todo ello se ha realizado bajo el sistema operativo de robots ROS con la ayuda de paquetes estándard. El correcto funcionamiento de este esquema local de Localización y Modelado Simultáneos (SLAM) se ha comprobado experimentalmente sobre terreno inclinado.
\end{abstract}

Palabras clave: Robots móviles, telémetro láser, nube de puntos 3D, SLAM local.

\section{INTRODUCCIÓN}

Los robots móviles terrestres necesitan disponer de información detallada y actualizada del entorno que los rodea para poder navegar de forma segura. Los telémetros láser tridimensionales (3D) son sensores que se utilizan con asiduidad para obtener nubes de puntos de la superficie por la que se debe desplazar el vehículo. Así, resulta frecuente emplearlos sobre terreno irregular en áreas de catástrofes [8], zonas naturales [13] o superficies planetarias [11].

Muchos de los sensores láser 3D utilizados en robótica se construyen añadiendo una rotación a un telémetro láser comercial bidimensional (2D). Este tipo de escáner es más económico, pero el tiempo de adquisión de un barrido láser $3 \mathrm{D}$ denso resulta bastante significativo [5]. Por ello, hasta no hace demasiado tiempo, era usual detener al robot para realizar la toma de datos 3D [9]. En la actualidad, para integrar sin detenerse todos los rangos medidos en un sistema de referencia común se requiere emplear algún método de Localización y Modelado Simultáneos (SLAM) [1][14]. La nube de puntos $3 \mathrm{D}$ resultante puede servir para repre- sentar el terreno cercano al robot móvil durante la navegación local [4].

Este artículo describe la adquisición de barridos 3D nivelados en movimiento con el robot móvil Andábata (ver Figura 1) bajo el sistema operativo para robots ROS [10]. El correcto funcionamiento de este esquema de SLAM local para corregir distorsiones debidas al desplazamiento del vehículo se ha comprobado de forma experimental a baja velocidad.

El artículo está organizado de la siguiente forma. En el apartado 2 se repasan las principales características de Andábata incluyendo su sensor láser 3D. A continuación se describen los nodos empleados en ROS y las relaciones entre los mismos. Los resultados experimentales se presentan en el apartado 4. Por último, se muestran las conclusiones obtenidas.

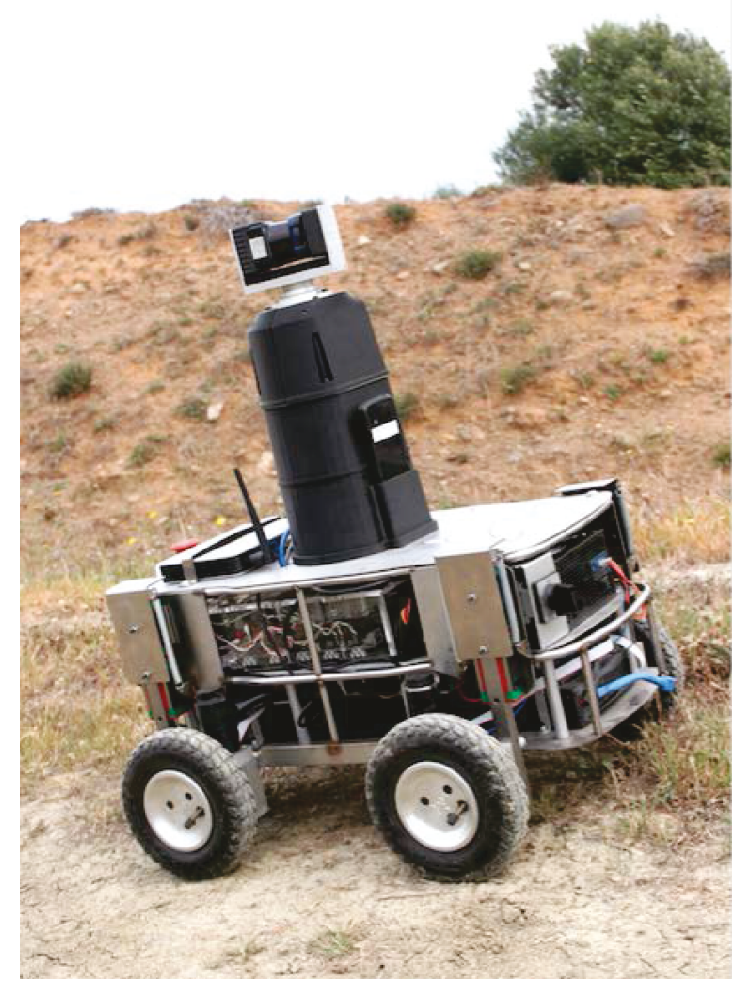

Figura 1: El robot móvil Andábata. 


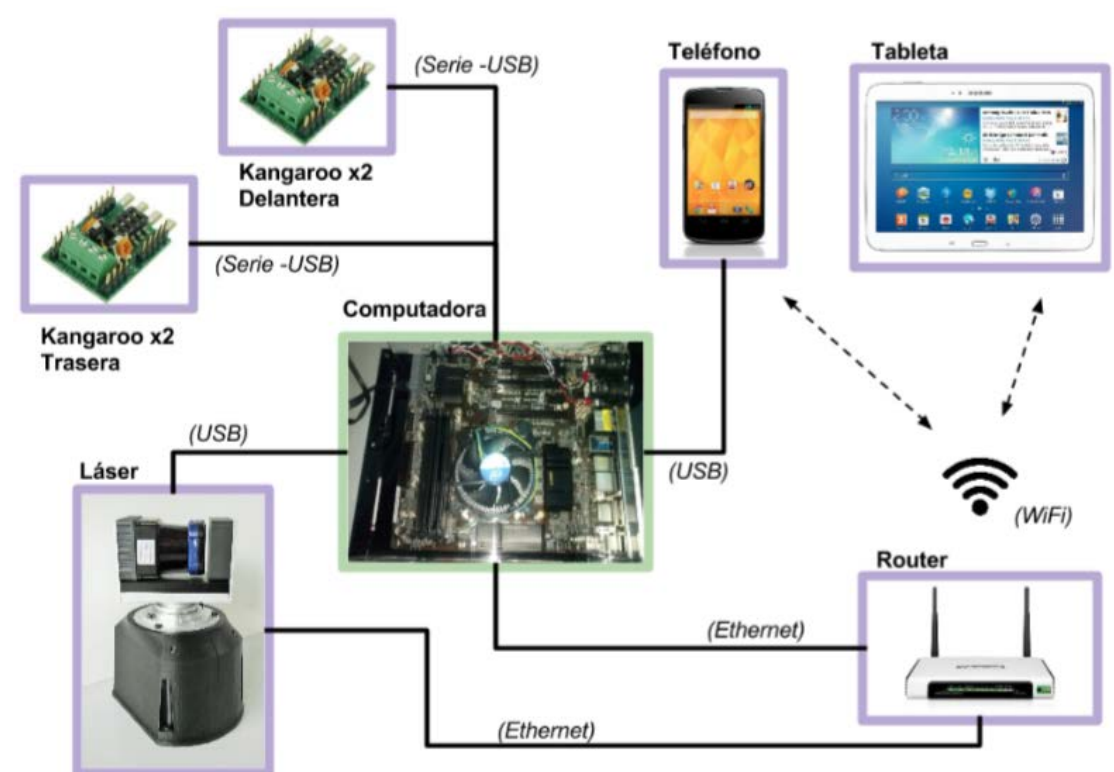

Figura 2: Conexiones entre los distintos elementos de Andábata.

\section{EL ROBOT MÓVIL ANDÁBATA}

Andábata es un robot móvil $4 \times 4$ alimentado por baterías construido para la navegación autónoma en entornos naturales [6]. El robot pesa $41 \mathrm{~kg}$ en total y sus dimensiones máximas son $67 \mathrm{~cm}$ de largo, $54 \mathrm{~cm}$ de ancho y $81 \mathrm{~cm}$ de alto (ver Figura 1).

El robot tiene tres niveles: el inferior en el que se sitúan las baterías, el intermedio para las etapas de potencia de los motores y la computadora, y el superior para los sensores y el enrutador WiFi. La computadora posee un procesador Intel Core i7 4771 (3,5 GHz, $8 \mathrm{MB}$ cache) y $16 \mathrm{~GB}$ RAM.

Andábata se desplaza mediante tracción por deslizamiento (skid-steering) que le proporcionan sus cuatro ruedas de $20 \mathrm{~cm}$ de diámetro. Cada rueda posee su propio motor con codificador, una reductora con relación de 1 a 37, y un sistema de suspensión pasiva formado por dos muelles y una guía lineal de $6,5 \mathrm{~cm}$ de recorrido. La velocidad máxima del vehículo es de $0,68 \mathrm{~m} / \mathrm{s}$ en línea recta, pero decrece conforme se reduce el radio de giro.

El control de bajo nivel de los motores de tracción se compone de dos etapas de potencia conmutadas Sabertooth 2x32 conectadas a dos placas de control Kangaroo x2. Estas últimas reciben de la computadora las órdenes de velocidad de las ruedas, y le envían las velocidades estimadas de cada rueda a través de un puerto USB (ver Figura 2).

Andábata cuenta con un teléfono móvil para obtener datos de su GPS, inclinómetros, giróscopos y magnetómetros. El teléfono también envía imáge- nes de vídeo a una tableta para la supervisión remota del robot mediante el enrutador WiFi (ver Figura 2).

Además, se ha construido un telémetro láser 3D, llamado UNO-Motion, a partir del escáner bidimensional (2D) Hokuyo UTM-30LX-EW. El sensor $3 \mathrm{D}$ pesa $1,9 \mathrm{~kg}$ y sus dimensiones son $125 \times$ $170 \times 122 \mathrm{~mm}$ [5]. Tiene una cabeza giratoria, donde se ubica el telémetro láser 2D y una base que proporciona el giro adicional (ver Figura 3). La transmisión de energía y señales entre la base y la cabeza giratoria se realiza mediante un anillo rozante.

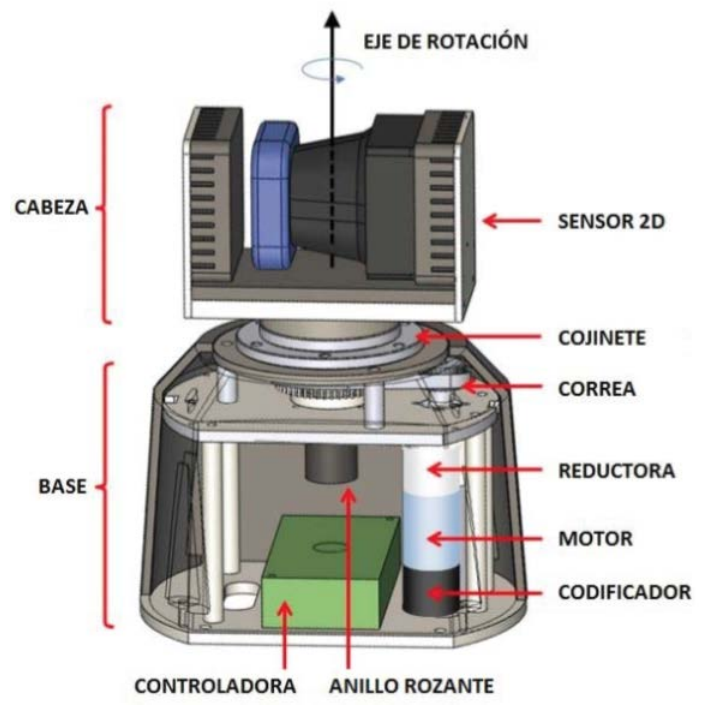

Figura 3: Principales componentes del escáner láser 3D. 
El eje de giro está alineado con el centro óptico del sensor 2D, por lo que el sensor 3D tiene el mismo rango de medidas de $0,1 \mathrm{~m}$ a $30 \mathrm{~m}$ (que se reduce a $15 \mathrm{~m}$ con luz solar directa) y la misma resolución angular vertical de $0,25^{\circ}$ que el escáner de Hokuyo. La resolución horizontal puede modificarse actuando sobre la velocidad de giro de la cabeza, de la que depende la velocidad de adquisición de un barrido 3D. Por ejemplo, para una resolución horizontal de $0,67^{\circ}$ se necesitan $6,75 \mathrm{~s}$ para completar un barrido $3 \mathrm{D}$ con media vuelta de la cabeza.

Además, la zona ciega del sensor $2 \mathrm{D}$ de $90^{\circ}$ se sitúa debajo para evitar interferencias con la base del sensor 3D. Así, la zona ciega de UNO-Motion es un cono en el que el radio de la base $h$ es igual a la altura del centro óptico del escáner (ver Figura 4). Montado sobre Andábata, se ha estimado una altura sobre el suelo de $h=0,723 \mathrm{~m} \mathrm{[5]}$.

\section{IMPLEMENTACIÓN EN ROS}

Sea $X Y Z$ el sistema de referencia asociado al robot situado en el centro geométrico del rectángulo definido por los puntos de contacto de las ruedas con el suelo. El eje $X$ está alineado con la dirección longitudinal del vehículo y su sentido de avance, mientras que el eje $Z$ es normal al plano de apoyo y apunta hacia arriba (ver Figura 5).

Las coordenadas cartesianas de los puntos de un barrido 3D van a estar expresadas en un sistema de referencia $X_{p} Y_{p} Z_{p}$ cuyo origen coincide con el del vehículo al inicio del barrido. El eje $Z_{p}$ se define con la misma dirección que la gravedad y sentido opuesto, el eje $X_{p}$ coincide con la proyección del eje $X$ sobre el plano horizontal, y el eje $Y_{p}$ se sitúa perpendicular al plano $Z_{p} X_{p}$ formando un sistema dextrógiro (ver Figura 5).

La Figura 6 muestra los nodos empleados en ROS para construir nubes de puntos 3D niveladas. En conjunto, se trata de un esquema de SLAM local a baja velocidad y sin cierre de bucles.

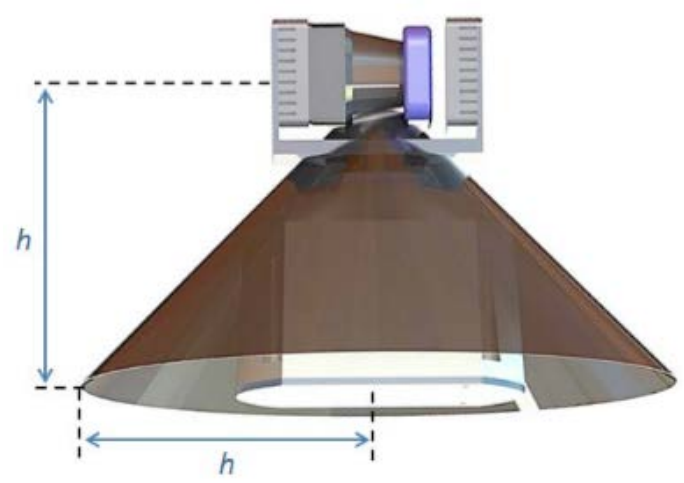

Figura 4: Zona ciega del telémetro láser 3D.

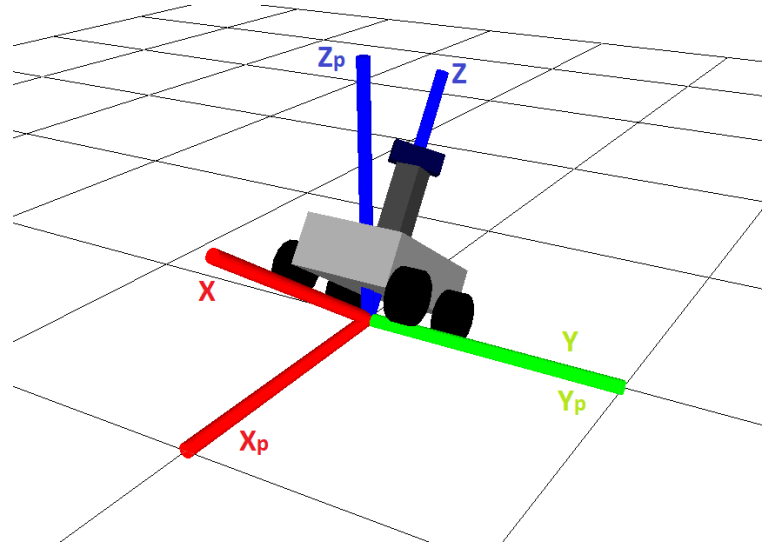

Figura 5: Sistemas de coordenadas del robot $(X Y Z)$ y del barrido nivelado $\left(X_{p} Y_{p} Z_{p}\right)$.

En primer lugar se puede observar el nodo teleoperación que envía consignas de velocidad a las ruedas del robot usando una tableta. Este nodo recibe información de dos aplicaciones desarrolladas en el sistema operativo Android:

- andabata, que es una adaptación de moverbot [2]. Esta aplicación se ejecuta tanto en la tableta, donde cuenta con una interfaz de usuario, como en el teléfono para comunicarse con la computadora a través de un puerto USB (ver Figura 2), y

- android_sensor_driver, que publica en la computadora topics con datos sensoriales de la tableta y del teléfono a través de la red WiFi [12].

El nodo odometría se encarga de computar la velocidad longitudinal y angular del vehículo. Para ello utiliza las medidas de las velocidades de las ruedas sobre un modelo cinemático 2D aproximado del sistema de locomoción [3].

El nodo robot_localizacion emplea un filtro de Kalman Unscented [7] para fusionar los ángulos de alabeo y cabeceo, la velocidad de guiñada del teléfono (que publica android_sensor_drivers), y las velocidades proporcionadas por odometría. De esta forma se obtiene la relación entre los sistemas de referencia $X_{p} Y_{p} Z_{p}$ y $X Y Z$.

Por su parte, el nodo uno_motion_node envía comandos de velocidad de giro y recibe de forma periódica (cada $25 \mathrm{~ms}$ ) el ángulo relativo entre la cabeza y la base al inicio de cada barrido 2D mediante la conexión USB del sensor 3D. Este nodo publica la transformación entre el sistema de referencia del sensor láser 2D y el del robot $X Y Z$. Además, el nodo urg_node permite configurar el sensor Hokuyo y publica los barridos 2D que la 


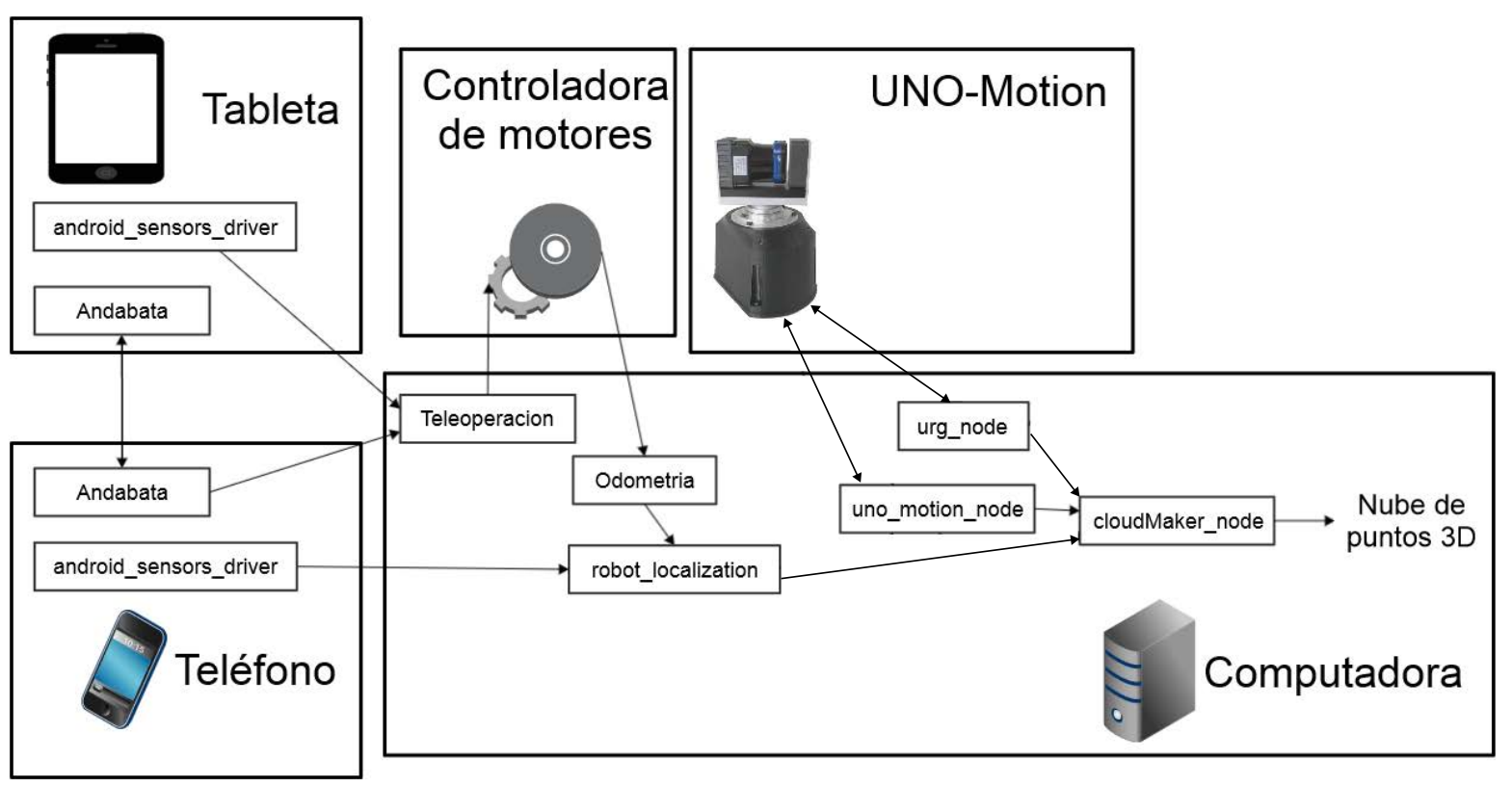

Figura 6: Nodos en ROS para implementar SLAM local.

computadora recibe a través de la conexión Ethernet (ver Figura 2).

Por último, el nodo cloudMaker_node publica la nube de puntos 3D nivelada combinando los barridos $2 \mathrm{D}$ mediante las transformaciones relativas entre el sistema de referencia del láser y el del vehículo $(X Y Z)$, y entre este último y el del barrido $3 \mathrm{D}\left(X_{p} Y_{p} Z_{p}\right)$.

\section{RESULTADOS EXPERIMENTALES}

Para comprobar el correcto funcionamiento del sistema de adquisición de datos programado en ROS, se han hecho experimentos en movimiento sobre terreno inclinado mientras Andábata se teleoperaba.

En la Figura 7 se muestran detalles de dos barridos $3 \mathrm{D}$ tras un giro de $180^{\circ}$ y de $360^{\circ}$ del sensor láser 2D. En ambos casos se puede observar la zona ciega alrededor del robot móvil y que hay zonas que se han muestreado de forma redundante. Sin embargo, con media vuelta de la cabeza del escáner $3 \mathrm{D}$ no se alcanza a observar todo el terreno alrededor del robot debido al propio movimiento del vehículo, por lo que resulta necesario dar una vuelta entera a la cabeza para poder tener una imagen completa.

En el vídeo que acompaña a este artículo se muestra la superposición de sucesivos barridos láser 3D en el sistema de referencia del primer barrido. Estos barridos completos se han obtenido en una carretera en pendiente durante un recorrido de ida y vuelta a baja velocidad (ver Figura 8(b)). Se puede observar que todos los barridos láser forman una imagen nítida del entorno cercano donde son claramente distinguibles los contornos de las barandillas y del edificio cercano (ver Figura 8(a)). De esta forma, se comprueba que el SLAM local no solo es válido para obtener por separado barridos láser 3D nivelados y sin distorsiones, sino que también puede funcionar bien para pequeños recorridos.

\section{CONCLUSIONES}

En este artículo se ha descrito el sistema de adquisición de barridos 3D nivelados con el robot móvil Andábata en movimiento. Este esquema de SLAM local se ha programado con una serie de nodos bajo el sistema operativo de robots ROS con la ayuda de paquetes estándard. Para ello, se integran cada uno de los barridos 2D con la información odométrica e inercial, y las medidas de inclinación del vehículo para producir coordenadas Cartesianas niveladas referenciadas al inicio del barrido. De esta forma, se corrigen las distorsiones en la toma de datos debidas al movimiento del vehículo.

El correcto funcionamiento de todos los nodos desarrollados en ROS se ha comprobado experimentalmente con Andábata sobre terreno inclinado. En la actualidad, los barridos láser 3D nivelados se están utilizando para la construcción de mapas locales con los que se planifican caminos para la navegación autónoma en entornos naturales mediante el GPS y el magnetómetro del teléfono móvil. 


\section{Agradecimientos}

Este trabajo ha sido subvencionado en parte por el proyecto español DPI 2015-65186-R y por el proyecto andaluz PE-2010 TEP-6101-R.

\section{Referencias}

[1] H. Almqvist, M. Magnusson, and A. Lilienthal, Improving point cloud accuracy obtained from a moving platform for consistent pile attack pose estimation, Journal of Intelligent and Robotic Systems: Theory and Applications, vol. 75, no. 1, pp. 101-128, 2014.

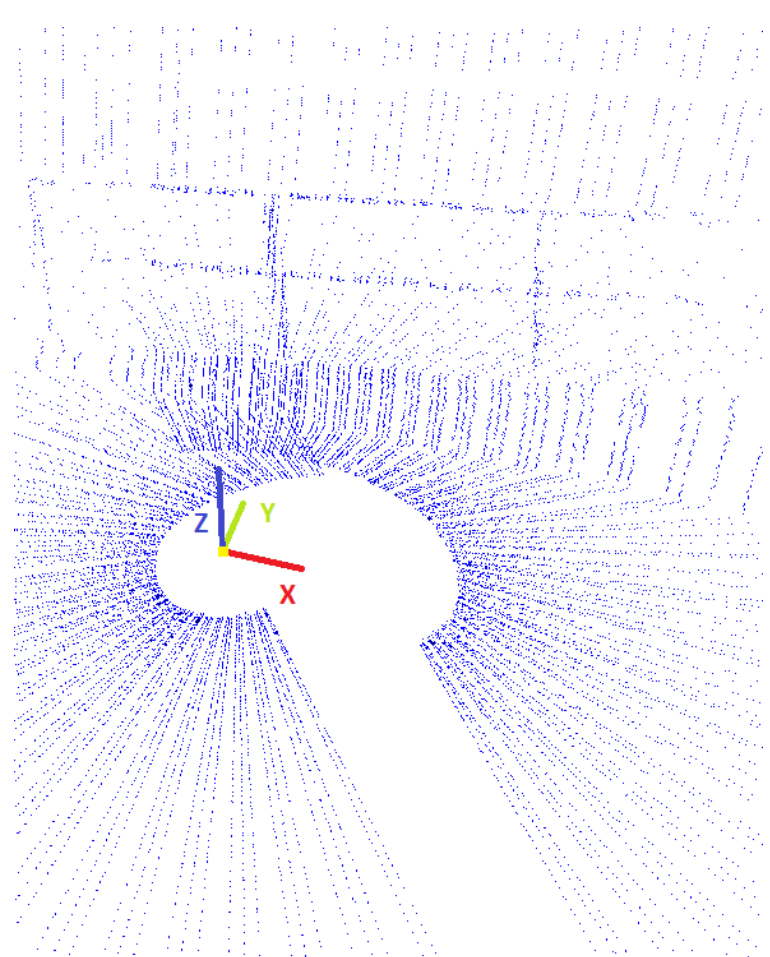

(a)

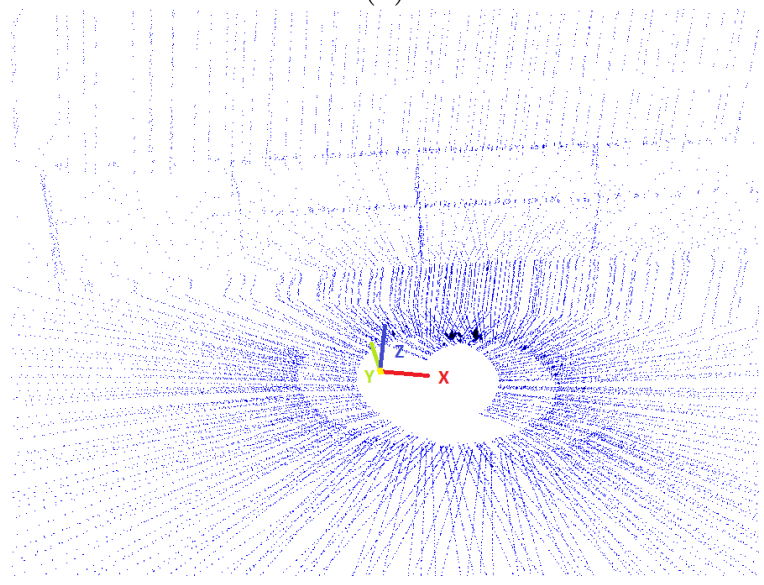

(b)

Figura 7: Detalle de dos barridos láser 3D obtenidos tras media vuelta (a) y tras una vuelta completa (b) del sensor láser 2D.
[2] P. Bovbel and F. Andargie, Mover-bot, https://code.google.com/p/mover-bot/, 2014.

[3] A. Mandow, J. L. Martínez, J. Morales, J. L. Blanco, A. García-Cerezo and J. González Experimental kinematics for wheeled skid-steer mobile robots, Proc. IEEE International Conference on Intelligent Robots and Systems, San Diego, USA, pp. 1222-1227, 2007.

[4] J. L. Martínez, A. Mandow, A. Reina and T. J. Cantador, J. Morales and A. García-Cerezo, Navigability analysis of natural terrains with fuzzy elevation maps from ground-based $3 D$ range scans, Proc. IEEE/RSJ International Conference on Intelligent Robots and Systems, Tokyo, Japan, pp. 1576-1581, 2013.

[5] J. L. Martínez, J. Morales, A. J. Reina, A. Mandow, A. Pequeño-Boter and A. GarcíaCerezo, Construction and calibration of a lowcost $3 D$ laser scanner with $360^{\circ}$ field of view for mobile robots, in Proc. IEEE International Conference on Industrial Technology, Sevilla, pp. 149-154, 2015.

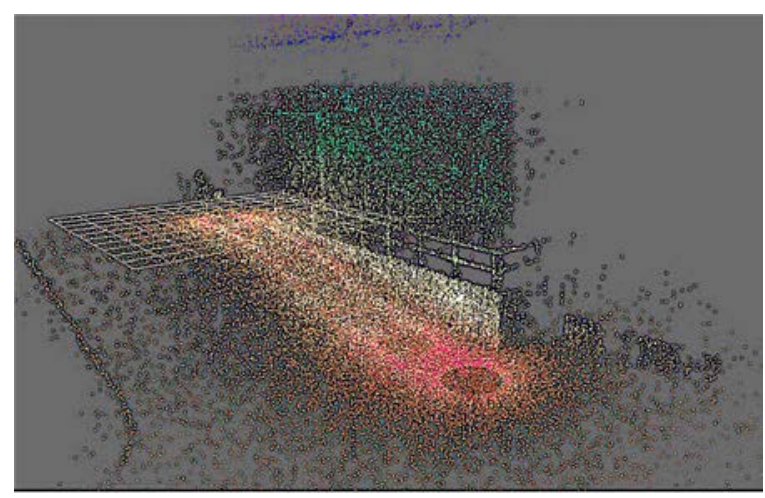

(a)

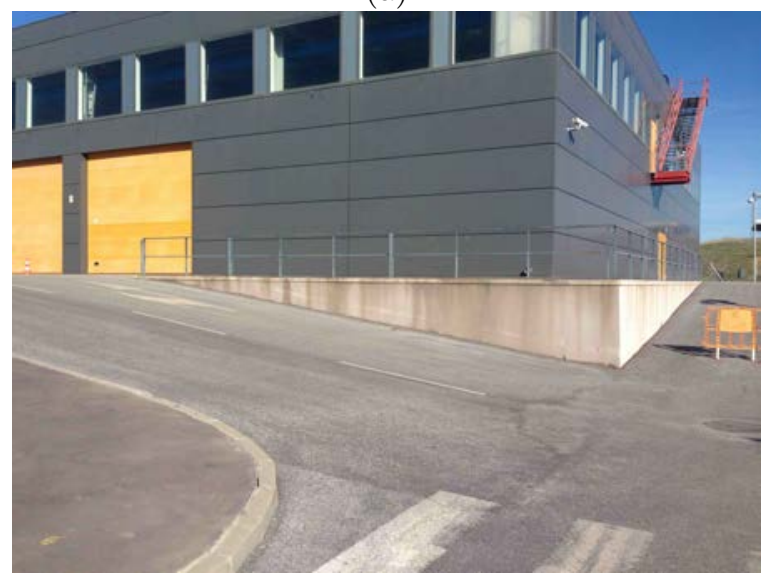

(b)

Figura 8: Superposición de sucesivos barridos 3D obtenidos en movimiento (a) sobre una carretera con pendiente (b). 
[6] J. L. Martínez, Navegación autónoma de un robot móvil $4 X_{4}$, http://www.uma.es/cms/base/ver/section/ document/73618/navegacion-autonoma-deun-robot-movil-4x4/, 2016.

[7] T. Moore and D. Stouch, A Generalized Extended Kalman Filter Implementation for the Robot Operating System, Proc. 13th International Conference on Intelligent Autonomous Systems, Padova, Italia, 2014.

[8] K. Nagatani, N. Tokunaga, Y. Okada, K. Yoshida, Y. Hada, T. Yoshida and E. Koyanagi, Teleoperation of all-terrain robot using continuous acquisition of three-dimensional environment under time-delayed narrow bandwidth communication, Proc. IEEE International Workshop on Safety, Security \& Rescue Robotics, Denver, USA, 2009.

[9] A. Nüchter, 3D Robotic Mapping: The Simultaneous Localization and Mapping Problem with Six Degrees of Freedom, Chapter 6: Experiments and Results, Springer-Verlag, 2009.
[10] M. Quigley, B. Gerkey, K. Conley, J. Faust,T. Foote, J. Leibs, E.Berger, R. Wheeler, and A. $\mathrm{Ng}$, ROS: an open-source robot operating system, Proc. Workshop on Open Source Software, IEEE International Conference on Robotics and Automation, Kobe, Japan, 2009.

[11] I. Rekleitis, J. L. Bedwani, E. and Dupuis, T. Lamarche and P. Allard,Autonomous overthe-horizon navigation using LIDAR data, Autonomous Robots, vol. 34, num. 1-2, pp. 1-18, 2013.

[12] C. Rockey and A. Furlan, ROS Android Sensors Driver, https://github.com/chadrockey/android_sen sors_driver, 2012.

[13] J. Serón, J. L. Martínez, A. Mandow, A. J. Reina, J. Morales, and A. García-Cerezo, Automation of the arm-aided climbing maneuver for tracked mobile manipulators, IEEE Transactions on Industrial Electronics, vol. 61, no. 7, pp. 3638-3647, 2014.

[14] R. Zlot and M. Bosse, Efficient large-scale three-dimensional mobile mapping for underground mines, Journal of Field Robotics, vol. 31, no. 5, pp. 731-752, 2014. 